\title{
Brazilin Suppresses Inflammation via the Down-regulation of IRAK4 in LPS-stimulated Raw264.7 Macrophage
}

\author{
Kye-Yoon Yoon ${ }^{1, \#}$, Kui-Jin Kim ${ }^{1, \#}$, Hyung-Sun Youn ${ }^{2}$, Sei-Ryang Oh$^{3}$, Boo-Yong Lee ${ }^{1, *}$ \\ ${ }^{1}$ Department of Food Science and Biotechnology, CHA University, Seongnam, South Korea \\ ${ }^{2}$ Department of Biomedical Laboratory Science, Soon Chun Hyang University, Asan, South Korea \\ ${ }^{3}$ Natural Medicine Research Center, Korea Research Institute of Bioscience and Biotechnology, Cheongju, South Korea \\ "These authors contributed equally to this work. \\ *Corresponding author: bylee@cha.ac.kr
}

\begin{abstract}
Brazilin, is a bioactive compound extracted from Caesalpinia sappan Linn, has been reported the protective effect of the immune system. Particular attention is now devoted to better understanding of the molecular basis of bazilin anti-inflammatory activity. In the present study, we studied the effect of brazilin on the Raw264.7 macrophage cell lines by a nutrigenomics approaches. Raw264.7 cells were treated with braziln, then treated with LPS to cause inflammation. The nuclear transcription $\mathrm{\kappa B}(\mathrm{NF}-\mathrm{\kappa B})$ promoter activity were analyzed with dual luciferase assay kit. The gene expression and production levels of pro-inflammatory cytokine interleukin (IL)-1 $\beta$, tumor necrosis factor (TNF) $\alpha$, and IL-6 were evaluated with semi-quantitative RT-PCR and with ELISA, respectively. We also examined inflammatory signaling, including mitogen-activated protein kinase (MAPK) pathway, iNOS, COX2, and IRAK4. Our findings demonstrated that brazilin down-regulated the expression of IRAK4 protein lead to suppress of c-Jun $\mathrm{NH}_{2}$ terminal kinase (JNK) signaling, and subsequently inactivation of nuclear transcription $\kappa \mathrm{B}(\mathrm{NF}-\mathrm{\kappa B})$, inducible nitric oxide synthase (iNOS) and cyclooxygenase 2 (COX2) thus promoting the expression of the downstream target pro-inflammatory cytokines such as IL-1 $\beta$, TNF $\alpha$ and IL-6 in LPS stimulated Raw264.7 macrophage cell. Thus, brazilin showed anti-inflammatory activity in Raw264.7 macrophage cell targeting IRAK4 mediated signaling pathway.
\end{abstract}

Keywords: Brazilin, inflammation, NF- $\kappa B$, IRAK4, MAPK

Cite This Article: Kye-Yoon Yoon, Kui-Jin Kim, Hyung-Sun Youn, Sei-Ryang Oh, and Boo-Yong Lee, "Brazilin Suppresses Inflammation via the Down-regulation of IRAK4 in LPS-stimulated Raw264.7 Macrophage.” Journal of Food and Nutrition Research, vol. 3, no. 9 (2015): 575-580. doi: 10.12691/jfnr-3-9-3.

\section{Introduction}

Inflammation process is mainly associated with host organisms against exogenous pathogen and incidence of body injuries in healthy condition [1,2]. In contrast, stimulation of inflammation has been recognized as the integral feature of the chronic disease, which includes obesity, diabetes, cancer, and cardiovascular diseases $[3,4,5]$. Therefore, coordination of inflammatory response results in ameliorating inflammation-associated chronic disease.

Macrophage plays an important role in inflammation and in response to a variety of inflammatory mediators such as lipopolysaccharide and other exogenous products [6]. Toll-like receptors (TLRs) bind to highly conserved leucine-rich repeated sequences expressed by bacterial and viral pathogen-associated molecular patterns (PAMP) [7] and subsequently induce the release the pro-inflammatory cytokines through the myeloid differential factor (MyD88dependent) and toll-interleukin-1 receptor domaincontaining adapter inducing interferon- $\beta$ (TRIF-dependent) pathways $[8,9]$. The pro-inflammatory cytokines such as IL-1 $\beta$, TNF $\alpha$, and IL-6 play an important role in inflammation, promoting to induce other proinflammatory cytokines, chemokines, and somatic pain $[10,11]$, Cellular responses to pro-inflammatory cytokines depends on multiple protein kinase cascades including activation of the stress-activated mitogen activated protein kinase (MAPK), c-Jun $\mathrm{NH}_{2}$-terminal kinase (JNK), cascade of intracellular kinase (ERK), and p38 MAPK as well as transcription factor nuclear factor $\kappa \mathrm{B}(\mathrm{NF}-\kappa \mathrm{B})$ $[12,13]$.

The IL-1 receptor-associated kinase 4 (IRAK4) has been shown to play an important role in MyD88 dependent inflammatory signaling. Moreover, IRAK4 leads to increase of MAPK signaling and I $\kappa$ B kinase, and subsequently activation of $\mathrm{NF}-\kappa \mathrm{B}$ thus promoting the expression of the downstream target pro-inflammatory cytokines. While, the IRAK4 kinase deficient mice have been shown to be resistant to LPS stimulated inflammation, due to diminish MyD88-IRAK4 association and decrease the pro-inflammatory cytokine productions [14]. Therefore, suppression of IRAK4 is important for prevention of LPS-mediated inflammation. 
Brazilin is a major active phenolic component that is isolated from the heartwood of Caesalpinia sappan Leguminosae (C. sappan). C. sappan extracts has been used traditional complementary medicine as antiinflammation $[15,16]$. Brazilin has been reported several beneficial biological activities, including modulation of immune function and cancer progression [17,18,19]. However, how brazilin regulates inflammation in macrophage cell still remain unclear. Therefore, this study was performed to determine the molecular mechanism of braziln on LPS-induced inflammation in macrophage cell. To determine the effect of brazilin on LPS-mediated cellular inflammation, we used Raw264.7 macrophage cell. We analyzed the effect of brazilin on IRAK4 and its downstream targets including iNOS, COX2, NF- $\mathrm{B}$, MAPKs and pro-inflammatory cytokines in LPS-induced Raw264.7 macrophage cell.

\section{Materials and Methods}

\subsection{Materials}

Lipopolysaccharide (LPS; Escherichia coli 0111:B4) was purchased from Sigma (St. Louis, Mo, USA). Brazilin was obtained from dried C. sappan L. heartwood (purchased in Seoul, South Korea) methanol extracts, and was purified according to the methods described by $\mathrm{Oh}$ et al. [20]. The chemical structure of brazilin was identified by the Korea Research Institute of Bioscience \& Biotechnology as shown in Figure 1A. The extract was dissolved in dimethyl sulfoxide prior to use. All reagents were purchased from Sigma unless otherwise described.

A

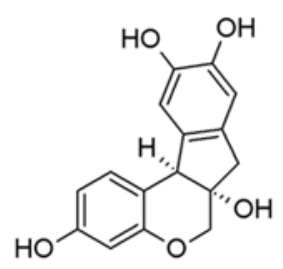

the cell viability analysis, Raw264.7 cells were treated with $0,10,30,50$, and $100 \mu \mathrm{M}$ of brazilin.

\subsection{Western Blotting}

Cells were plated overnight in 6-well plates at a density of $0.7 \times 10^{6}$ per plate, and further incubated in $1 \mu \mathrm{g} / \mathrm{mL}$ LPS for $1 \mathrm{~h}$ after treatment. Cells were harvested with icecold RIPA buffer (50mM Tris-HCl, 1mM EDTA, 1mM EGTA, 150mM NaCl, 1\% NP-40, 0.1\% sodium dodecyl sulfate (SDS), $0.25 \%$ sodium deoxycholate, and $5 \% \beta$ mercaptoethanol) with protease and phosphatase inhibitors. Cell lysates were centrifuged at $12,000 \times g$ for 5 min at $4^{\circ} \mathrm{C}$ and the supernatants were then collected. Protein concentrations were determined using the Bradford Protein Assay (Bio-Rad, Richmond, CA, USA). Equal amounts of extracted protein $(100 \mu \mathrm{g})$ were separated by SDS-polyacrylamide gel electrophoresis (SDS-PAGE) and transferred to polyvinylidene difluoride membranes. Membranes were blocked with 5\% non-fat skim milk phosphate-buffered saline (PBS) containing $0.05 \%$ Tween 20, and were blotted with the indicated primary antibody overnight at $4{ }^{\circ} \mathrm{C}$. Bound primary antibodies were detected with a peroxidase-coupled secondary antibody. The reactive bands were visualized by chemiluminescence (Amersham Biosciences, Piscataway, USA).

\subsection{RNA Extraction and Reverse Transcription Polymerase Chain Reaction (RT-PCR)}

Total RNA was extracted from RAW264.7 cells using Trizol ${ }^{\circledR}$ reagent (Invitrogen Corporation, Carlsbad, CA, USA), according to the manufacturer's protocol. Total RNA $(1 \mu \mathrm{g})$ was reverse transcribed to cDNA using the Maxime RT PreMix kit (Intron, Seongnam, South Korea). cDNA was then amplified with an Inno Hot Tap polymerase Kit (Bookyoung SM, South Korea). The primer sequences were as follows: GAPDH, forward (5'AACTTTGGCATTGTGGAAGG -3') and antisense (5'ACACATTGGGGGTAGGAACA-3'); TNF $\alpha$, forward (5'- CTACTCCTCAGAGCCCCCAG -3') and reverse (5'- TGACCACTCTCCCTTTGCAG -3'); IL-1 $\beta$, forward (5'-CAGGATGAGGACATGAGCACC-3') and reverse (5'-CTCTGCACACTCAAACTCCAC-3'); IL-6, forward (5'-GTTCTCTGGGAAATCGTGGA-3') and reverse (5'TGTACTCCAGGTAGCTA -3'). PCR amplification of the resulting cDNA template was conducted under the following conditions. PCR products were analyzed on 1\% agarose gels and stained with ethidium bromide. Images were captured with a Gene Fresh ultraviolet (UV) detector (Syngene Bio Imaging, South Korea). Densitometric analysis was performed using the ImageJ program (National Institutes of Health, Bethesda, MD). The results are representative of three independent experiments.

\subsection{Transient Transfection and Luciferase Assays}

For transfection, cells were seeded at a density of $7 \times 10^{4}$ cells/well in 48-well plates and incubated for $24 \mathrm{~h}$ until approximately $70-80 \%$ confluence. NF- $\kappa \mathrm{B}(2 \times)$-luciferase and COX-2 luciferase reporter plasmids or the corresponding empty vector plasmids were co-transfected 4-nitro-5-sulfophenyl)-2H-tetrazolium-5-carboxanilide (XTT) assay was performed as described by Kim et al [21]. For 
to Raw264.7 macrophage cell using SuperFect transfection reagent (Qiagen, Valencia, CA, USA), according to the manufacturer's instructions. Cells were lysed and luciferase activity was determined using the Promega luciferase assay system (Promega, Madison, CA, USA) and a luminometer (Perkin Elmer Cetus, Foster City, CA, USA). Luciferase activity was normalized to that of $\beta$-galactosidase.

\subsection{Nitrite Colorimetric Assay}

Raw264.7 cells were seeded at $7 \times 10^{4}$ per well in 96well plates and incubated for $24 \mathrm{~h}$. The cells were pretreated with various concentration of brazilin for $1 \mathrm{~h}$ and then treated with LPS $(1 \mu \mathrm{g} / \mathrm{mL})$ for an additional $24 \mathrm{~h}$. For nitrite determinations, $100 \mu \mathrm{L}$ of culture supernatant was mixed with an equal volume of Griess reagent and the absorbance at $540 \mathrm{~nm}$ was measured. The $\mathrm{NaNO}_{2}$ standard curve was used to determine total nitrite.

\subsection{Enzyme-linked Immunosorbent Assay (ELISA)}

Cells were pretreated with various brazilin concentrations for $1 \mathrm{~h}$ and then further stimulated with LPS $(1 \mu \mathrm{g} / \mathrm{mL})$ for $24 \mathrm{~h}$. The supernatants were collected and stored at $-80^{\circ} \mathrm{C}$ until cytokine analysis. IL- $1 \beta, \mathrm{TNF} \alpha$, and IL-6 levels in supernatants were determined using ELISA MAX ${ }^{\mathrm{TM}}$ Kits (BioLegend, San Diego, CA, USA), according to the manufacturer's instructions.

\subsection{Statistical Analysis}

All values are expressed as mean \pm standard deviation. Data were analyzed by one-way analysis of variance (ANOVA) (9.01, SAS program). Post-hoc comparisons were conducted using Tukey's honestly significant difference test. Differences were considered to be statistically significant when $p$ values were less than 0.05 .

\section{Results}

\subsection{Brazilin Decreased NF- $\kappa B$ Luciferase Activity in LPS-stimulated Raw264.7 Macrophage Cells}

The chemical structure of brazilin is presented in Figure 1A. The effect of brazilin on Raw264.7 cell viability was measured by a XTT assay. As shown in Figure 1B, brazilin decreased cell viability at a concentration of 100 $\mu \mathrm{M}$. Thus, the concentration of 10,30 , and $50 \mu \mathrm{M}$ brazilin was selected for the further investigation.

The induction of inflammatory response is modulated through the activation of NF- $\mathrm{BB}$. Therefore, we evaluated whether brazilin regulates the transcriptional activity of NF- $\kappa$ B. The Raw264.7 macrophage cells were transiently transfected with pNF-kB-Luc plasmid, pre-treated with different concentration of brazilin for $1 \mathrm{~h}$ and then cotreated with brazilin and LPS. NF- $\mathrm{BB}$ transactivation was measured by a dual luciferase assay system. Brazilin significantly suppressed LPS-induced transcriptional activity of NF- $\kappa \mathrm{B}$ compared to LPS-treated Raw264.7 with absence of brazilin as shown in Figure $1(\mathrm{p}<0.05)$.
We found that brazilin may show anti-inflammatory

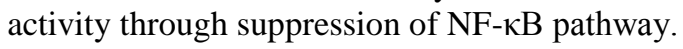

\subsection{Brazilin Inhibited the Upstream Target of NF-kB in LPS-stimulated Raw264.7 Macrophage Cell}

A number of genes involved in pro-inflammatory response are governed by NF- $\kappa \mathrm{B}$. To better evaluate the anti-inflammatory activity of brazilin on the transcription of NF- $\mathrm{NB}$ target gene, the protein level of iNOS and COX2, which depends on NF- $\mathrm{B}$ activity in response to LPS stimulation was investigated. As shown in Figure 2, western blotting analysis showed that LPS caused the elevated expression levels of iNOS and COX2, while both iNOS and COX2 proteins were significantly decreased in Raw264.7 with presence of brazilin.

\section{$1 \mu \mathrm{g} / \mathrm{mL}$ LPS}

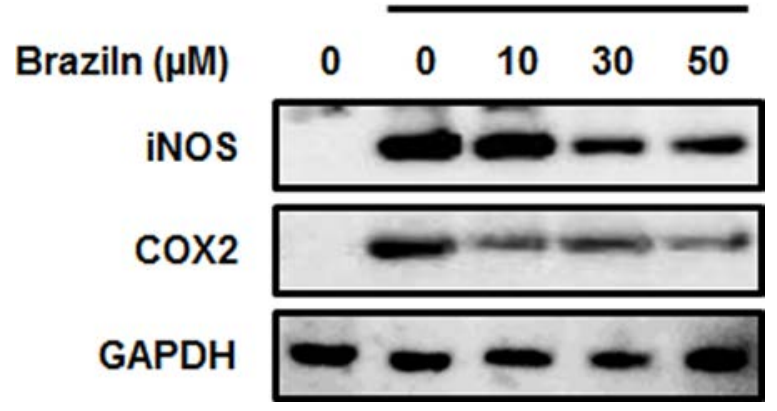

Figure 2. Brazilin down-regulated the expression of iNOS and COX2 protein in LPS stimulated Raw264.7. Raw 264.7 macrophage cells $\left(5 \times 10^{5} /\right.$ well $)$ were pretreated with 10,30 , or $50 \mu \mathrm{M}$ brazilin for $1 \mathrm{~h}$ and then induced with $1 \mu \mathrm{g} / \mathrm{mL}$ LPS for an $24 \mathrm{~h}$. The expression of iNOS and COX2 protein were detected by western blotting using iNOS, COX2, and GAPDH antibodies. DMSO was used as a vehicle. Data are expressed as mean $\pm \operatorname{SD}(n=3)$
A

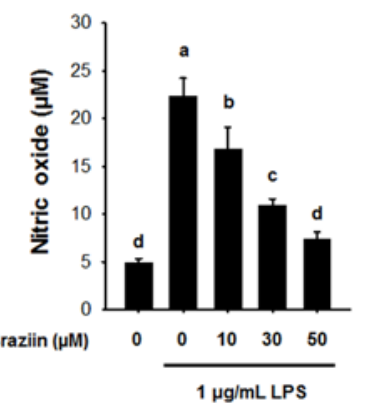

B

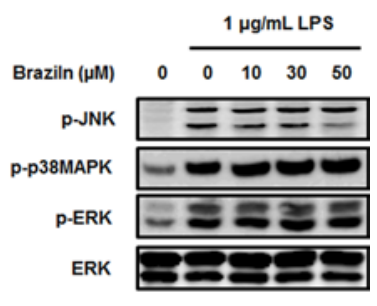

Figure 3. Brazilin decreased the nitric oxide production and phosphorylation of JNK in LPS-stimulated Raw264.7 macrophage cell. (A) The production of soluble nitrite oxide. Culture media was subsequently isolated and nitrite concentrations determined. (B) The expression of p-JNK, p-p38MAPK, pERK, and ERK in LPS-stimulated Raw264.7 macrophage cell. Cells were pretreated with 10,30 or $50 \mu \mathrm{M}$ brazilin for $1 \mathrm{~h}$ and then stimulated with $1 \mu \mathrm{g} / \mathrm{mL} \mathrm{LPS} \mathrm{for} 30 \mathrm{~min}$ or $24 \mathrm{~h}$. DMSO was used as a vehicle. Equal amounts of protein in cell lysates were analyzed by Western blot. Data are mean \pm SD $(n=3)$

\subsection{The Effect of Brazilin on ROS Production and MAPK Signaling in LPS- Stimulated Raw264.7 Macrophage Cell}

ROS and MAPK signaling pathway is crucial role for

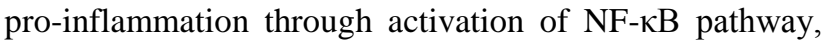


we examined the intracellular levels of NO using a nitrite colorimetric assay in LPS-induced Raw264.7 with the presence or absence of baseline. As shown in Figure 3A, LPS treatment caused significantly elevated the production of NO levels. However, pre-treatment of brazilin resulted in a decrease of LPS-induced NO levels in Raw264.7 macrophage cell. In addition, we determine the phosphorylation of the MAPK pathway, which are intermediate stage controlling of NF- $\kappa \mathrm{B}$ activation. As shown in Figure 3B, the phosphorylation of JNK was decreased by brazilin, but not in the phosphorylation of p38MAPK and ERK.

\section{$1 \mu \mathrm{g} / \mathrm{mL}$ LPS}

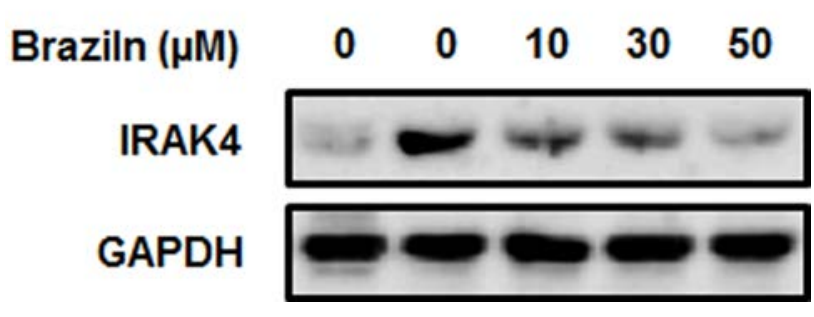

Figure 4. LPS increased the expression of IRAK4 protein, whereas brazilin diminished the expression of IRAK4 in Raw264.7 macrophage cell. Cells were pretreated with 10,30 or $50 \mu \mathrm{M}$ brazilein for $1 \mathrm{~h}$ and then stimulated with $1 \mu \mathrm{g} / \mathrm{mL}$ LPS for $30 \mathrm{~min}$ or $24 \mathrm{~h}$. DMSO was used as a vehicle. Equal amounts of protein in cell lysates were analyzed by Western blot

A

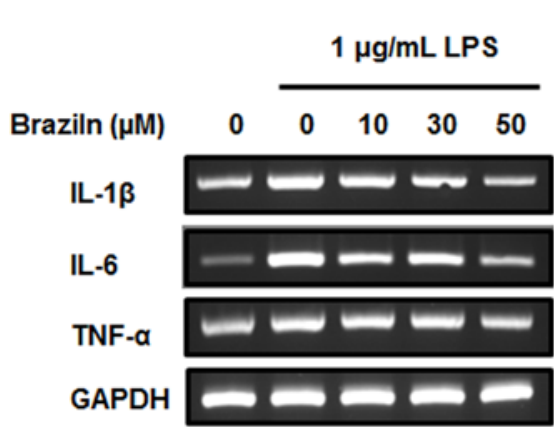

C

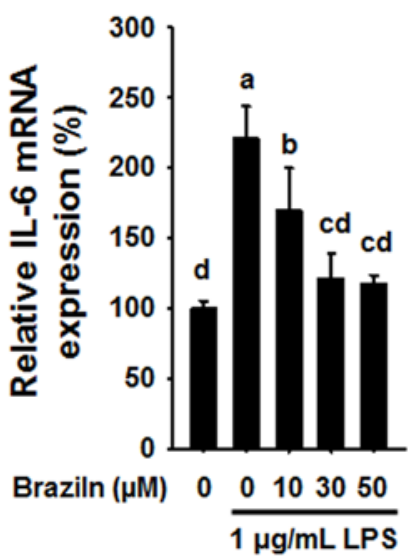

In fact, IRAK4 is known as the upstream enzymes responsible for phosphorylating MAPK [9]. Next, we determined the expression levels of IRAK4, which are major mediators controlling the NF- $\mathrm{KB}$ activation in LPSinduced inflammation. As shown in Figure 4, we sought that brazilin suppressed the expression of IRAK4 in a dosed dependent manner in LPS-induced Raw264.7 compared to the vehicle-treated and LPS-induced Raw264.7 macrophage cell. These results suggest that brazilin could attenuate LPS-induced inflammation in Raw264.7 macrophage, in part, by inhibition of IRAK4 regulated NF- $\kappa \mathrm{B}$ activation, and partially affecting the MAPK pathway.

\subsection{Brazilin Attenuates mRNA Expression of Pro-inflammatory Cytokines in LPS-Stimulated Raw264.7 Macrophage Cell}

The pro-inflammatory cytokines is en end product of IRAK4 regulated NF- $\kappa \mathrm{B}$ activation and play critical roles in the extent of inflammation. To finalize the analysis of the brazilin on anti-inflammatory activity, we measured the expression of pro-inflammatory cytokine mRNA and the levels of cytokine production in LPS-induced Raw264.7 macrophage cell with presence of absence of brazilin. For this objective, the expression levels of proinflammatory transcripts were analyzed by semiquantitative RT-PCR. The pro-inflammatory cytokine was measured by ELISA.

B

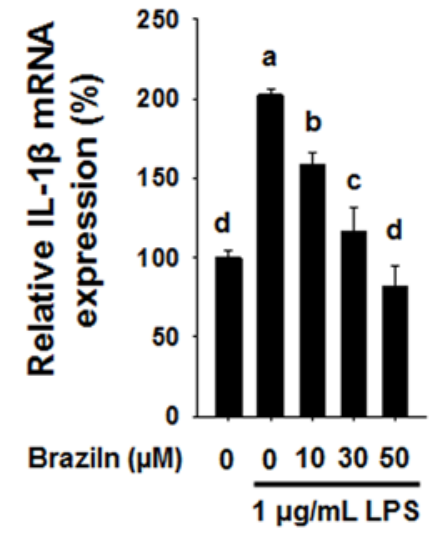

D

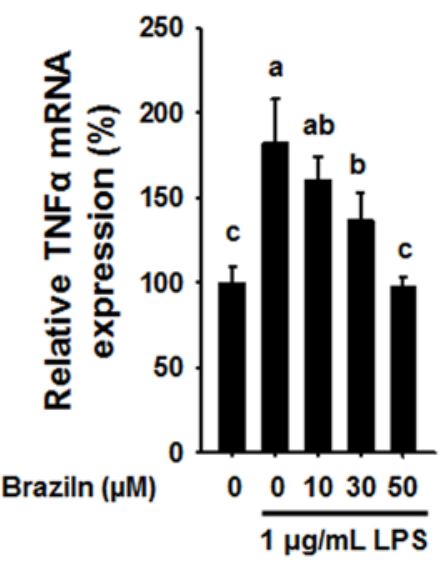

Figure 5. The transcription levels of pro-inflammatory cytokines were down-regulated by brazilin in LPS-induced Raw264.7. Cells were pretreated with 10,30 , or $50 \mu \mathrm{M}$ brazilein for $1 \mathrm{~h}$, stimulated with LPS $(1 \mu \mathrm{g} / \mathrm{mL})$, and incubated for a further $24 \mathrm{~h}$. DMSO was used as a vehicle. Data are expressed as mean \pm SD $(n=3)$. (A) Total RNA was amplified by RT-PCR using the indicated primers for IL-1 $\beta$, TNF $\alpha$, and IL-6. (B-D) The relative mRNA expression of IL-1 $\beta, \mathrm{TNF} \alpha$, and IL-6.were quantified using the ImageJ program. Data are mean \pm SD $(n=3)$ 
As shown in Figure 5A-5D, brazilin decreased the expression of genes encoding IL-1 $\beta, \mathrm{TNF} \alpha$, and IL-6 in a dose dependent manner in LPS-treated Raw264.7 macrophage cell. Moreover, pro-inflammatory cytokine production including IL- $1 \beta$ and TNF $\alpha$ were decreased by 30 and $50 \mu \mathrm{M}$ brazilin compared to LPS-induced Raw264.7 macrophage cell as shown in Figure 6A and 6B. In particular, brazilin clearly depressed IL-6 production in LPS-induced Raw264.7 macrophage cell in a dosedependent manner as shown in Figure 6C. These results indicated that baseline could regulate IRAK4-mediated $\mathrm{NF}-\kappa \mathrm{B}$ activation pro-inflammatory signaling in LPSinduced Raw264.7 macrophage cell.

\section{Discussion}

Several studies showed that the isomer of brazilin such as brazilein exhibited inflammation suppressive activity and anti-oxidative function [6,22]. However, the molecular targets of brazilin for inflammation response have remained elusive. In this study, we focused on the identification of the molecular target of brazilin and quantification of its effect on anti-inflammation in LPSinduced Raw264.7 macrophage cell.

A previous study demonstrates that TLR/IRAK4 signaling enhances macrophage-associated inflammation [23] and IRAK4 deficient animal model are completely resistant to high dose of LPS [24]. Our results showed that LPS caused not only the pro-inflammatory cytokine production, but also increase of NF- $\mathrm{NB}$ promoter activity via activation of IRAK4 protein activity, while brazilin dramatically repressed the expression levels of IRAK4 and subsequently decreased NF- $\kappa B$ activity. NF- $\mathrm{B}$ signaling is required for the transcription of iNOS and COX2, which are thought to be expressed mainly by activated macrophage $[25,26]$. We also found that brazilin inhibited the expression of $\mathrm{NF}-\mathrm{\kappa B}$ dependent protein COX2 and iNOS levels as well as their product NO in LPS-induced Raw264.7 macrophage cell.

In addition, stimulation of LPS promotes to release the serum pro-inflammatory cytokines IL-1 $\beta$, IL-6, and TNF $\alpha$ in animal model $[27,28]$. Treatment of LPS increased the production of IL-1 $\beta$, IL-6, and TNF $\alpha$ by Raw264.7 macrophage cell in mouse serum. We observed that LPSinduced production of IL-1 $\beta$, IL-6, and TNF $\alpha$ were markedly decreased in Raw264.7 macrophage cell with presence of brazilin. In fact, we and others showed that bioactive compounds, such as bebeerine, fucoidan, and hispidin suppressed the production of pro-inflammatory cytokines and subsequent ameliorated inflammation response which is associated with aberrant expression of MAPK signaling [29,30,31].

MAPK signaling promote to enhance the phosphorylation of NF- $\kappa \mathrm{B}$, which induces the function of $\mathrm{NF}-\kappa \mathrm{B}$ in nucleus [32]. Indeed, bioactive compound shows anti-inflammatory activities through the suppression of p38 MAPK, JNK, and ERK mediated NF-אB pathway in macrophage [29,30,33,34]. Similarly, we found that brazilin significantly reduced the phosphorylation of JNK in a dose dependent manner. However, p38MAPK and ERK were not altered protein expression after brazilin treatment. This result raised the indication that braziln regulated the phosphorylation of JNK and subsequently

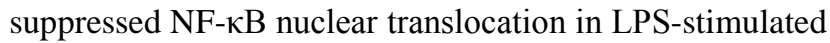
Raw264.7 macrophage cell.

\section{Conclusion}

We provide that brazilin decreased the expression of IRAK4 levels lead partially to suppression of MAPK signaling involving JNK, which resulted in inhibition of $\mathrm{NF}-\kappa \mathrm{B}$ activity that is changing the transcription of iNOS and COX2 and subsequently attenuate the inflammatory response end products such as pro-inflammatory cytokines and nitrites. Our data provide proof that brazilin suppress a major source of inflammatory mediators and that macrophage cells are highly responsive to ameliorate a variety of inflammatory factors. Therefore, we suggest that brazilin might be a useful bioactive compound for the prevention of exogenous pathogen-mediated diseases as well as IRAK4-associated inflammatory diseases.

\section{Conflict of Interest}

The authors declare that they have no conflict of interest.

\section{References}

[1] Munford RS, Pugin J. Normal responses to injury prevent systemic inflammation and can be immunosuppressive. American journal of respiratory and critical care medicine 2001; 163:316-21.

[2] Mookherjee N, Brown KL, Bowdish DM, Doria S, Falsafi R, Hokamp K, et al. Modulation of the TLR-mediated inflammatory response by the endogenous human host defense peptide LL-37. J Immunol 2006; 176:2455-64.

[3] Xu H, Barnes GT, Yang Q, Tan G, Yang D, Chou CJ, et al. Chronic inflammation in fat plays a crucial role in the development of obesity-related insulin resistance. J Clin Invest 2003; 112:1821-30.

[4] Dandona P, Aljada A, Bandyopadhyay A. Inflammation: the link between insulin resistance, obesity and diabetes. Trends Immunol 2004; 25:4-7.

[5] Chen R, Alvero AB, Silasi DA, Mor G. Inflammation, cancer and chemoresistance: taking advantage of the toll-like receptor signaling pathway. American journal of reproductive immunology 2007; 57:93-107.

[6] Bae IK, Min HY, Han AR, Seo EK, Lee SK. Suppression of lipopolysaccharide-induced expression of inducible nitric oxide synthase by brazilin in RAW 264.7 macrophage cells. Eur J Pharmacol 2005; 513:237-42.

[7] Newton K, Dixit VM. Signaling in innate immunity and inflammation. Cold Spring Harbor perspectives in biology 2012; 4.

[8] Lu YC, Yeh WC, Ohashi PS. LPS/TLR4 signal transduction pathway. Cytokine 2008; 42:145-51.

[9] Akira S, Takeda K. Toll-like receptor signalling. Nat Rev Immunol 2004; 4:499-511.

[10] Borovikova LV, Ivanova S, Zhang M, Yang H, Botchkina GI, Watkins LR, et al. Vagus nerve stimulation attenuates the systemic inflammatory response to endotoxin. Nature 2000; 405:458-62.

[11] Sommer C, Kress M. Recent findings on how proinflammatory cytokines cause pain: peripheral mechanisms in inflammatory and neuropathic hyperalgesia. Neuroscience letters 2004; 361:184-7.

[12] Herlaar E, Brown Z. p38 MAPK signalling cascades in inflammatory disease. Mol Med Today 1999; 5:439-47.

[13] Tak PP, Firestein GS. NF-kappaB: a key role in inflammatory diseases. J Clin Invest 2001; 107:7-11.

[14] Li X. IRAK4 in TLR/IL-1R signaling: possible clinical applications. European journal of immunology 2008; 38:614-8.

[15] Zhao H, Bai H, Wang Y, Li W, Koike K. A new homoisoflavan from Caesalpinia sappan. Journal of natural medicines 2008; 62:325-327. 
[16] NAMIKOSHI M, NAKATA H, YAMADA H, NAGAI M, SAITOH T. Homoisoflavonoids and related compounds. II. Isolation and absolute configurations of 3, 4-dihydroxylated homoisoflavans and brazilins from Caesalpinia sappan L. Chemical and pharmaceutical bulletin 1987; 35:2761-2773.

[17] Gao XJ, Wang TC, Zhang ZC, Cao YG, Zhang NS, Guo MY. Brazilin plays an anti-inflammatory role with regulating Toll-like receptor 2 and TLR 2 downstream pathways in Staphylococcus aureus-induced mastitis in mice. Int Immunopharmacol 2015; 27:130-7.

[18] Jeon J, Lee JH, Park KA, Byun HS, Lee H, Lee Y, et al. Brazilin selectively disrupts proximal IL-1 receptor signaling complex formation by targeting an IKK-upstream signaling components. Biochem Pharmacol 2014; 89:515-25.

[19] Kim B, Kim SH, Jeong SJ, Sohn EJ, Jung JH, Lee MH, et al. Brazilin induces apoptosis and G2/M arrest via inactivation of histone deacetylase in multiple myeloma U266 cells. Journal of agricultural and food chemistry 2012; 60:9882-9.

[20] Oh SR, Kim DS, Lee IS, Jung KY, Lee JJ, Lee HK. Anticomplementary activity of constituents from the heartwood of Caesalpinia sappan. Planta Med 1998; 64:456-8.

[21] Kim KJ, Lee OH, Lee BY. Fucoidan, a sulfated polysaccharide, inhibits adipogenesis through the mitogen-activated protein kinase pathway in 3T3-L1 preadipocytes. Life sciences 2010; 86:791-7.

[22] Ye M, Xie WD, Lei F, Meng Z, Zhao YN, Su H, et al. Brazilein, an important immunosuppressive component from Caesalpinia sappan L. Int Immunopharmacol 2006; 6:426-32.

[23] Koziczak-Holbro M, Joyce C, Gluck A, Kinzel B, Muller M, Tschopp C, et al. IRAK-4 kinase activity is required for interleukin-1 (IL-1) receptor- and toll-like receptor 7-mediated signaling and gene expression. J Biol Chem 2007; 282:13552-60.

[24] Suzuki N, Suzuki S, Duncan GS, Millar DG, Wada T, Mirtsos C, et al. Severe impairment of interleukin-1 and Toll-like receptor signalling in mice lacking IRAK-4. Nature 2002; 416:750-6.

[25] Suh N, Honda T, Finlay HJ, Barchowsky A, Williams C, Benoit $\mathrm{NE}$, et al. Novel triterpenoids suppress inducible nitric oxide synthase (iNOS) and inducible cyclooxygenase (COX-2) in mouse macrophages. Cancer research 1998; 58:717-23.

[26] Blanco AM, Pascual M, Valles SL, Guerri C. Ethanol-induced iNOS and COX-2 expression in cultured astrocytes via NF-kappa B. Neuroreport 2004; 15:681-5.

[27] Olson JK, Miller SD. Microglia initiate central nervous system innate and adaptive immune responses through multiple TLRs. J Immunol 2004; 173:3916-24.

[28] Blanque R, Meakin C, Millet S, Gardner CR. Hypothermia as an indicator of the acute effects of lipopolysaccharides: comparison with serum levels of IL1 beta, IL6 and TNF alpha. General pharmacology 1996; 27:973-7.

[29] Shao HJ, Jeong JB, Kim KJ, Lee SH. Anti-inflammatory activity of mushroom-derived hispidin through blocking of NF-kappaB activation. Journal of the science of food and agriculture 2015; 95:2482-6.

[30] Kim KJ, Yoon KY, Lee BY. Low molecular weight fucoidan from the sporophyll of Undaria pinnatifida suppresses inflammation by promoting the inhibition of mitogen-activated protein kinases and oxidative stress in RAW264.7 cells. Fitoterapia 2012; 83:1628-35.

[31] Jeong HW, Hsu KC, Lee JW, Ham M, Huh JY, Shin HJ, et al. Berberine suppresses proinflammatory responses through AMPK activation in macrophages. American journal of physiology Endocrinology and metabolism 2009; 296:E955-64.

[32] Schulze-Osthoff K, Ferrari D, Riehemann K, Wesselborg S. Regulation of NF-kappa B activation by MAP kinase cascades. Immunobiology 1997; 198:35-49.

[33] Ryu SJ, Choi HS, Yoon KY, Lee OH, Kim KJ, Lee BY. Oleuropein suppresses LPS-induced inflammatory responses in RAW 264.7 cell and zebrafish. Journal of agricultural and food chemistry 2015; 63:2098-105.

[34] Huang GJ, Huang SS, Deng JS. Anti-inflammatory activities of inotilone from Phellinus linteus through the inhibition of MMP-9, NF-kappaB, and MAPK activation in vitro and in vivo. PloS one 2012; 7:e35922. 